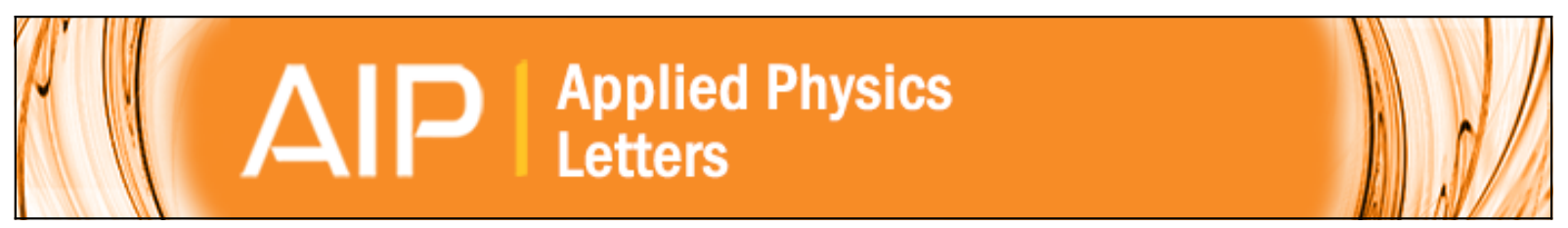

\title{
The effect of passivation on different GaAs surfaces
}

Ted H. Yu, Liang Yan, Wei You, Ramesh B. Laghumavarapu, Diana Huffaker, and Christian Ratsch

Citation: Applied Physics Letters 103, 173902 (2013); doi: 10.1063/1.4826480

View online: http://dx.doi.org/10.1063/1.4826480

View Table of Contents: http://scitation.aip.org/content/aip/journal/apl/103/17?ver=pdfcov

Published by the AIP Publishing

\section{Articles you may be interested in}

Dual passivation of GaAs (110) surfaces using $\mathrm{O} 2 / \mathrm{H} 2 \mathrm{O}$ and trimethylaluminum

J. Chem. Phys. 139, 244706 (2013); 10.1063/1.4852155

First-principles study of $\mathrm{GaAs}(001)$ - $\beta 2$ ( $2 \times 4$ ) surface oxidation and passivation with $\mathrm{H}, \mathrm{Cl}, \mathrm{S}, \mathrm{F}$, and $\mathrm{GaO}$ J. Appl. Phys. 107, 103720 (2010); 10.1063/1.3369540

Effects of Si deposition on the properties of Ga-rich (4×6) GaAs (001) surfaces

J. Vac. Sci. Technol. B 21, 1945 (2003); 10.1116/1.1585075

Surface and bulk passivation of GaAs solar cell on Si substrate by $\mathrm{H} 2+\mathrm{PH} 3$ plasma

Appl. Phys. Lett. 76, 730 (2000); 10.1063/1.125876

The origin of $\mathrm{Ga} 2 \mathrm{O} 3$ passivation for reconstructed $\mathrm{GaAs}(001)$ surfaces

J. Appl. Phys. 83, 5880 (1998); 10.1063/1.367449

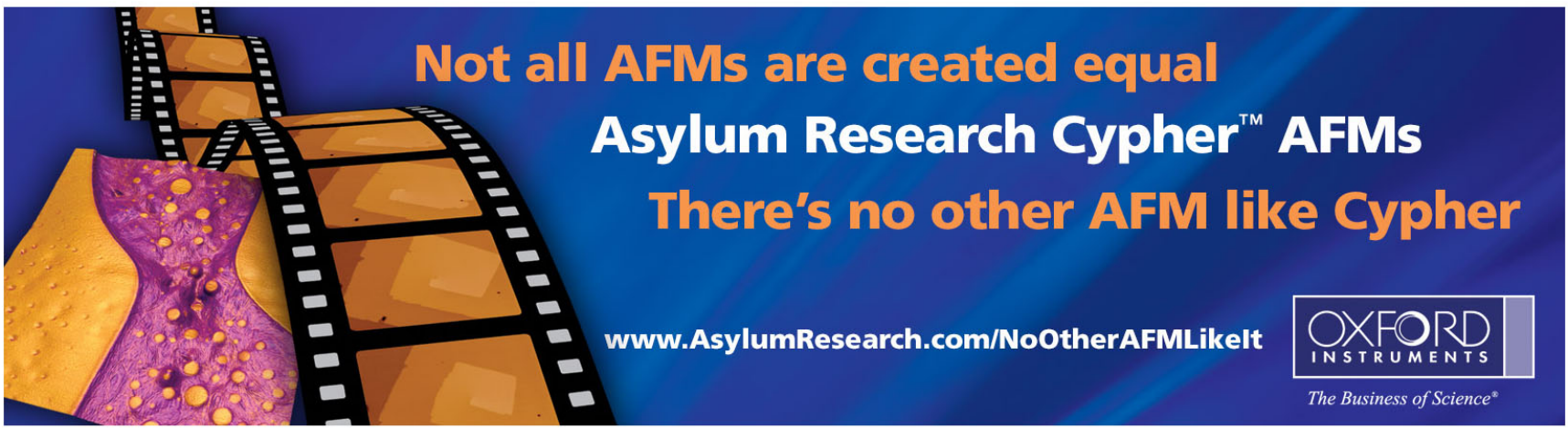




\title{
The effect of passivation on different GaAs surfaces
}

\author{
Ted H. Yu, ${ }^{1,2, a)}$ Liang Yan, ${ }^{3}$ Wei You, ${ }^{3}$ Ramesh B. Laghumavarapu, ${ }^{4}$ Diana Huffaker, ${ }^{4}$ \\ and Christian Ratsch ${ }^{1}$ \\ ${ }^{1}$ Department of Mathematics, Institute of Pure and Applied Mathematics, University of California \\ at Los Angeles, Los Angeles, California 90095, USA \\ ${ }^{2}$ Department Chemical Engineering, California State University Long Beach, Long Beach, California 90840 , \\ USA \\ ${ }^{3}$ Department of Chemistry, University of North Carolina, Chapel Hill, Chapel Hill, North Carolina 27599 , \\ USA \\ ${ }^{4}$ Department of Electrical Engineering, University of California at Los Angeles, Los Angeles, \\ California 90095, USA
}

(Received 18 June 2013; accepted 2 October 2013; published online 22 October 2013)

\begin{abstract}
The surface passivation of semiconductors on different surface orientations results in vastly disparate effects. Experiments of GaAs/poly(3,4-ethylenedioxythiophene/indium tin oxide solar cells show that sulfur passivation results in threefold conversion efficiency improvements for the GaAs (100) surface. In contrast, no improvements are observed after passivation of the GaAs (111B) surface, which achieves $4 \%$ conversion efficiency. This is explained by density-functional theory calculations, which find a surprisingly stable (100) surface reconstruction with As defects that contains midgap surface states. Band structure calculations with hybrid functionals of the defect surface show a surface state on the undimerized As atoms and its disappearance after passivation. (C) 2013 AIP Publishing LLC. [http://dx.doi.org/10.1063/1.4826480]
\end{abstract}

III-V semiconductors are attractive materials that are used in a variety of cutting-edge devices, such as solar cells. A fundamental aspect of these devices is their limitation at the interface because of surface states. It is important to understand the nature of these surface states, which may be caused by adatoms, surface defects, or the interface between two materials. Such an understanding will lead to better methods to reduce surface states through surface passivation. Recent reports have demonstrated dramatic improvements in solar cell efficiency after surface passivation. ${ }^{1-6}$ Unpassivated surface states lead to a recombination of photogenerated charge carriers, which lowers energy conversion rates. A popular passivation method is sulfur surface passivation ${ }^{4,6-8}$ with $\mathrm{Na}_{2} \mathrm{~S} \cdot 9 \mathrm{H}_{2} \mathrm{O},{ }^{8}$ ammonium sulfide, $\left(\mathrm{NH}_{4}\right)_{2} \mathrm{~S} /\left(\mathrm{NH}_{4}\right)_{2} \mathrm{~S}_{\mathrm{x}},{ }^{4,6,9,10}$ or thiols. ${ }^{11,12}$ In addition to increasing solar cell efficiencies, sulfur passivation improves photoluminescence intensity, ${ }^{8,12}$ makes the Schottky barrier height dependent on the contact metal, ${ }^{9}$ improves the $\mathrm{C}-\mathrm{V}$ characteristics of metal/insulator/semiconductor structures, ${ }^{10}$ and heightens the peak power output of lasers. ${ }^{13}$ While sulfur passivation has been demonstrated to improve device performances on the (100) surface, ${ }^{8-11,13}$ very few articles have demonstrated sulfur passivation of the (110) or (111) surfaces. Sulfur passivation removed native oxides in the GaAs (110) and (111) surfaces, ${ }^{14,15}$ but, oddly, these works did not report any device enhancement effects on these surfaces.

There is some ongoing debate on how passivation improves semiconductors. Earlier theoretical models ${ }^{7,16,17}$ looked at sulfur bonded GaAs (100) surfaces that have unique reconstructions at high temperature, such as the $2 \times 1$ reconstruction ${ }^{17}$ at $580^{\circ} \mathrm{C}$ (Refs. $14,18-20$ ) and the $2 \times 6$ reconstruction ${ }^{21}$ at $370^{\circ} \mathrm{C}$. Although these structures were found at high temperatures, sulfur passivation is typically a

a)Electronic Mail: ted.yu@csulb.edu room temperature process. ${ }^{4,6,8}$ As such, these unique surfacereconstructed structures of sulfur bonded GaAs are not the structures we consider in this letter.

More recent papers on sulfur passivation have pointed to the role of native oxides in the creation of surface states. This was originally proposed by Nannichi and co-workers, ${ }^{22}$ who explained why a $\left(\mathrm{NH}_{4}\right)_{2} \mathrm{~S}$ treatment improves GaAs devices in their experiments. Their model builds on Spicer's "unified defect model, ${ }^{23}$ a model which proposes that the midgap surface states can arise due to the formation of $\mathrm{Ga}$ or As defects, which in turn create dangling bonds on the surface. These dangling bond states lead to recombination losses in GaAs devices. Nannichi and co-workers explained that the defects appear when native oxides form on the GaAs surface. While most studies show agreement that midgap surface states arise by native oxides, previous density-functional theory (DFT) studies have explained the exact chemical nature of these surface states in two different ways. The first has attributed the midgap surface states intrinsically to bonds formed between the oxygen and the GaAs. ${ }^{24-27}$ The second has attributed the midgap surface states to Ga or As dangling bonds formed when the native oxides create defects on the GaAs surface, ${ }^{28-30}$ unrelated to bonds formed with oxygen.

In this Letter, we aim to shed some light on the nature of these surface states. We present experimental results as well as theoretical results based on DFT. The experimental results show that passivation is dependent on the surface orientation, which leads to improvements in conversion efficiency for the GaAs (100) surface but not for the GaAs (111B) surface. This is explained by our DFT results, which show that surface defects can form more easily on the (100) surface than on the (111B) surface. Because of the higher density of defect surface states on the GaAs (100) surface, passivation will have a dramatic effect on this surface orientation in comparison with the (111B) surface. We further show with 
TABLE I. Reported open circuit voltage $\left(\mathrm{V}_{\mathrm{oc}}\right)$, conversion efficiency $(\eta)$, and saturated current $\left(\mathrm{J}_{\mathrm{s}}\right)$, of four different ITO/PEDOT:PSS/GaAs Schottky junction solar cells. The effect of the passivation on the GaAs (100) Schottky junction is significant, while its effect on GaAs (111B) is not. The saturated current of the GaAs (111B) devices is lower than that of GaAs (100), which shows that there are fewer surface states on the (111B) surface.

\begin{tabular}{lcccc}
\hline \hline Surface & Passivation & $\mathrm{V}_{\mathrm{OC}}(\mathrm{V})$ & $\eta(\%)$ & $\mathrm{J}_{\mathrm{S}}\left(\mathrm{nA} / \mathrm{cm}^{2}\right)$ \\
\hline$(100)$ & No & 0.33 & 0.67 & 2.54 \\
$(100)$ & Yes & 0.51 & 1.91 & 0.93 \\
$(111 \mathrm{~B})$ & No & 0.65 & 4.08 & $1.3 \times 10^{-3}$ \\
$(111 \mathrm{~B})$ & Yes & 0.65 & 3.84 & $1.0 \times 10^{-3}$ \\
\hline \hline
\end{tabular}

theory that sulfur reacts with the most likely GaAs (100) surface defect to remove surface states.

Our experiments ${ }^{31}$ consist of conversion efficiency tests of inorganic/organic hybrid solar cell devices. The solar cell sandwiches consist of GaAs, PEDOT:PSS (poly(3,4-ethylenedioxythiophene: poly(styrenesulfonate)), and ITO (indium tin oxide) glass. We tested four different GaAs surfaces: (100) and (111B), with and without sulfur passivation. The sulfur passivation was a two-step process ${ }^{11}$ with octanethiol solution followed by ammonium sulfide solution in the layer between the GaAs and PEDOT:PSS. The PEDOT:PSS was spin coated on the passivated/nonpassivated GaAs and ITO glass. We brought the PEDOT:PSS sides together, while facing each other, to form the GaAs/PEDOT:PSS/ITO sandwich. All of the processes were performed at room temperature. The characteristics of the solar cells I-V were tested with dark and bright current measurements, which determined the $\mathrm{V}_{\mathrm{oc}}$, conversion efficiency, and saturated current as provided in Table I.

When we compare the efficiency, $\eta$, of the passivated versus unpassivated GaAs (100) device, we see in Table I that $\eta$ is dramatically improved with passivation by nearly a factor of three. The saturated current, $\mathrm{J}_{\mathrm{s}}$, is reduced from 2.54 to $0.93 \mathrm{~mA} / \mathrm{cm}^{2}$ after passivation. In addition, $V_{o c}$ is increased from 0.33 to $0.51 \mathrm{~V}^{31}$ These results suggest that the improvement is due to the reduction of surface states. For the GaAs (111B) surface, we see that there is little efficiency change from passivation. This is a highly reproducible result, and we consistently see $\sim 2-3$ times efficiency improvement for the (100) surfaces up to $\sim 2 \%$, while the efficiency of the (111B) surface stays relatively unchanged at $\sim 4 \%$ for many samples. The slight efficiency decrease of $0.24 \%$ for this particular (111B) sample may be a result of the thin sulfur passivation layer, which decreases light absorption.

In earlier works, it was found experimentally that sulfur passivation removes native oxides on GaAs (100), (110), and (111B) surfaces. ${ }^{14,15,20}$ For the (111B) surface, we find that the removal of these native oxides by sulfur passivation does not improve conversion efficiency. This result suggests that the bonds formed by the native oxides on the GaAs (111B) are not the direct cause of the midgap surface state, but rather these states are caused by surface defects which can remain even after the native oxides are removed. Our subsequent theoretical analysis from DFT explores how defects can cause surface states and explains the orientation dependence of sulfur passivation.
The FHI-AIMS ${ }^{32}$ code is used for the DFT calculations. It is an all-electron code that uses atom centered numeric orbitals as a basis set. For these calculations, we use the predefined light basis setting, which has radial $s, p$, and $d$ characters with an overall cutoff radius of $5 \AA$ and a Hartree potential expansion up to $l=4$. DFT with the Perdew-BurkeErnzerhof (PBE) ${ }^{33}$ approximation of the generalized gradient approximation (GGA) exchange-correlation functional and with accurate Van der Waals (VdW) corrections ${ }^{34}$ is used to relax the five bi-layered GaAs slabs with 10 bi-layers of vacuum. The bottom three bi-layers are fixed, and the bottom Ga layer of the GaAs slabs is terminated with pseudohydrogen atoms that contain 1.25 electrons to represent bonds to As atoms. The slab thickness, vacuum layer thickness, $\mathrm{k}$-points, and basis set are found to be well converged for the GaAs surface in terms of energy differences.

DFT with the Heyd-Scuseria-Ernzerhof (HSE-06) ${ }^{35}$ exchange-correlation functional and a non-optimized $25 \%$ mixing factor is used to determine the band structure and density of states (DOS) of the geometrically relaxed surfaces. This hybrid functional does not underestimate the band gap like the GGA functionals and in some cases is able to match the bulk band gap. When using experimental lattice parameter, a bulk band gap of $1.34 \mathrm{eV}$ is obtained, compared to the experimental value of $1.42 \mathrm{eV} .{ }^{36}$ Both the PBE-VdW and HSE-VdW calculations have an optimized GaAs lattice parameter of $5.71 \AA$, which is used in all calculations. This is only a $1 \%$ overestimation from the experimental lattice parameter of $5.65 \AA \AA^{37}$ Using the optimized lattice parameter, the most important GaAs $(100) \beta 2(2 \times 4)$ surface reconstruction on a five bi-layer slab is found to have a band gap of $1.65 \mathrm{eV}$. For GaAs slabs, the calculated band gap is highly thickness-dependent and decreases toward the bulk value as the slab thickness is increased beyond five bi-layers. ${ }^{25,38}$

Figure 1 shows our phase diagram results for the GaAs (100) surface using the method outlined previously. ${ }^{39,40}$ It includes a number of previously studied surface reconstructions and some defect surfaces. From our calculations, the

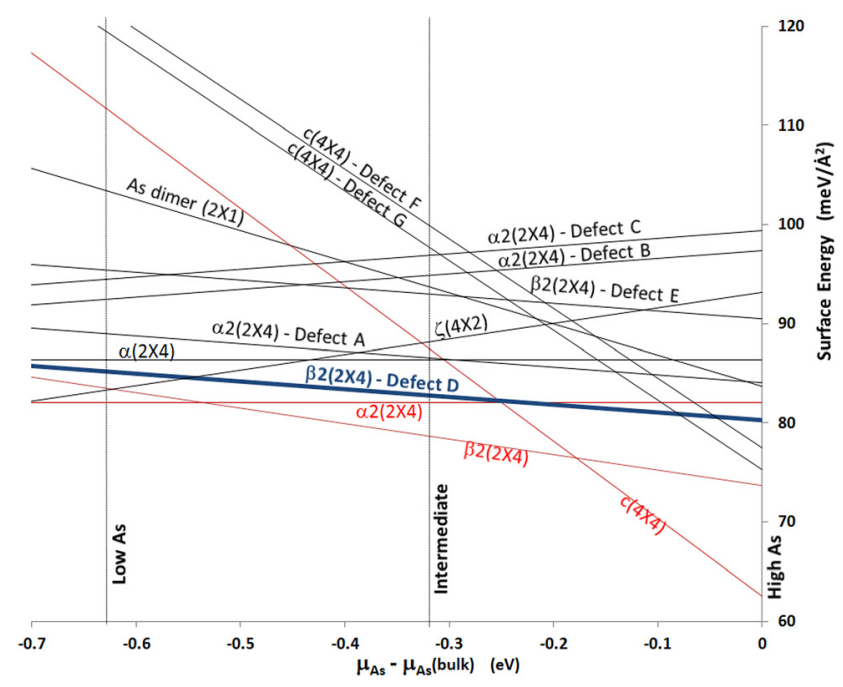

FIG. 1. Phase diagram for different GaAs (100) surface reconstructions. The three most stable reconstructions are (red lines) $\alpha 2(2 \times 4), \beta 2(2 \times 4)$, and $c(4 \times 4)$. Also represented are surface reconstructions with surface defects. The most stable defect surface reconstruction, $\beta 2(2 \times 4)$-Defect $D$ is shown in a bold blue line. 


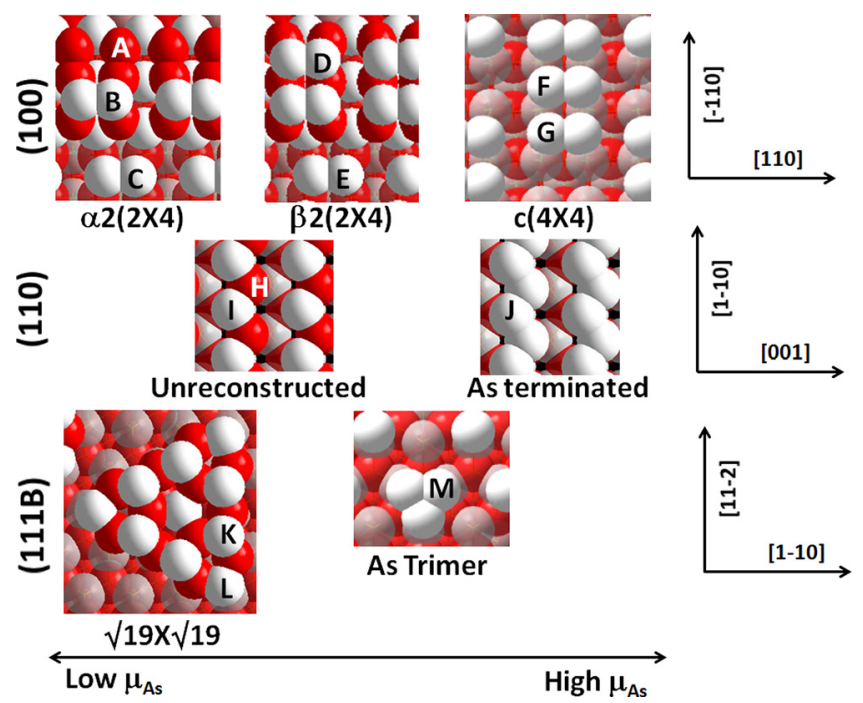

FIG. 2. As (white) and Ga (red) defects on GaAs reconstructed surfaces. The energy (in $\mathrm{eV}$ ) to remove an As or Ga from the surfaces at sites is listed in Table II. The lowest energy to remove a defect is in position D of the $\beta 2(2 \times 4)$ surface with an energy of $0.84 \mathrm{eV}$.

thermodynamically favorable surface reconstructions for GaAs $(100)$ are $\alpha 2(2 \times 4)$ at low $\mu_{\mathrm{As}}, \beta 2(2 \times 4)$ at intermediate $\mu_{\mathrm{As}}$, and $\mathrm{c}(4 \times 4)$ at high $\mu_{\mathrm{As}}{ }^{40}$ For the GaAs $(111 \mathrm{~B})$ surface, the favorable surface reconstructions are the $\sqrt{ } 19 \times \sqrt{ } 19$ reconstruction at low $\mu_{\mathrm{As}}$ and the As trimer $(2 \times 2)$ at intermediate and high $\mu_{\mathrm{As}}{ }^{40,41}$ For the GaAs (110) surface, the unreconstructed surface is the most stable, and at high $\mu_{\text {As }}$, the As-terminated surface reconstruction is most stable. ${ }^{40}$ These results agree with phase diagrams based on chemical potential reported previously. ${ }^{40,41}$ The only exception is that previous DFT results with PBE using pseudopotentials show that the GaAs $(100) \zeta(4 \times 2)$ reconstruction ${ }^{42}$ is most favorable at low $\mu_{\text {As }}$, while the all-electron code we use show that the $\alpha 2(2 \times 4)$ reconstruction is slightly more stable. ${ }^{43}$

While these surfaces are thermodynamically most favorable, we are interested in the energy cost to create defects on them. According to the paper by Nannichi and co-workers, ${ }^{22}$ the surface states arise when native oxides create defects of As or $\mathrm{Ga}$ on the GaAs surface. Therefore, the energy cost to create a defect of As or Ga from the GaAs surface will be a direct indicator of the native oxide's ability to create surface states. Figure 2 shows the possible As and Ga defect sites, and Table II shows the energies required to remove the surface atoms into a bulk phase reservoir, where $E_{n}$,defect $=E_{\text {defect surface }}+E_{n \text { (bulk) }}-E_{\text {perfect surface, }}$ and $n=$ As or Ga.

After removal of an As or Ga atom, the surface no longer satisfies the electron counting rule, ${ }^{44}$ and midgap surface states appear (see band structure calculations below). For the $\alpha 2(2 \times 4)$ surface, the lowest energy to create such a surface state is to remove a $\mathrm{Ga}$ atom at a cost of $0.88 \mathrm{eV}$. For the

TABLE II. Defect energies, $\mathrm{E}_{\mathrm{n}}$, defect, of various surface sites. The defect energy for site $\mathrm{D}$ is the lowest.

$\begin{array}{lllllllllllllll}\text { Site } & \text { A } & \text { B } & \text { C } & \text { D } & \text { E } & \text { F } & \text { G } & \text { H } & \text { I } & \text { J } & \text { K } & \text { L } & \text { M }\end{array}$

E (eV) $0.88 \begin{array}{llllllllllll}1.95 & 2.21 & \mathbf{0 . 8 4} & 2.15 & 1.91 & 1.63 & 1.82 & 1.77 & 1.16 & 1.75 & 1.75 & 1.45\end{array}$ $\beta 2(2 \times 4)$ reconstruction, the energy cost is $0.84 \mathrm{eV}$ to remove a top As atom. For the $c(4 \times 4)$ reconstruction, the energy cost is $1.63 \mathrm{eV}$ to remove an As atom from the corner. The reason that the energy cost of removing a top As atom in the $\beta 2(2 \times 4)$ reconstruction is so stable is because the two $\mathrm{Ga}$ atoms underneath form a stable $\mathrm{Ga}-\mathrm{Ga}$ bond after the removal of the As atom. This is similar to the Ga dimerization seen in the stable $\alpha 2(2 \times 4)$ reconstruction as reported originally by Yamaguchi. ${ }^{45}$ We find that for the GaAs (100) surface, the $c(4 \times 4)$ structure has the highest cost to create a midgap surface state and would be the most desirable structure to minimize surface states. Therefore, our calculations suggest that high $\mu_{\mathrm{As}}$ during the growth of the GaAs (100) will reduce surface states caused by As defects.

When comparing the (100) and (111B) GaAs surface orientations, we compare the $(100) \beta 2(2 \times 4)$ and $(111 \mathrm{~B})$ As trimer surfaces (which are stable at intermediate $\mu_{\mathrm{As}}$ ). Their energy costs for creating an As defect are $0.84 \mathrm{eV}$ and $1.45 \mathrm{eV}$, respectively. We find that for the GaAs (100) surface, the energy to create Defect $D$ is lower, and a surface state can be generated much easier than for the (111B) surface. We interpret our results as follows: the energy to remove an As atom to generate a defect on the GaAs (111B) surface is so large that the native oxides do not generate surface states on this surface, making the passivation step unnecessary, as is evident in the negligible conversion efficiency improvement in our experimental (111B) device upon passivation. In the phase diagram of Figure 1, we see that the surface structure $\beta 2(2 \times 4)$-Defect $D$ is the most stable defect structure of the GaAs (100) surface. We note that it is surprisingly more favorable than the $\alpha(2 \times 4)$ reconstruction. ${ }^{40}$

In Figure 3, we show the band structure and DOS of the $\beta 2(2 \times 4)$-Defect D surface. The DOS of a perfect $\beta 2(2 \times 4)$ surface (dashed line) has no surface states. Defect D generates a surface state, V1, and a conduction band minimum $(\mathrm{CBM})$ state, $\mathrm{C} 1$. Figure 4 shows the $\beta 2(2 \times 4)$ Defect D surface structure and the location of $\mathrm{V} 1$ and $\mathrm{C} 1$ orbitals. When the As defect is created, the bonds to atoms $\mathrm{A}, \mathrm{B}$, and $\mathrm{C}$ are broken. The atoms $\mathrm{B}$ and $\mathrm{C}$ dimerize, which stabilize this structure. This leaves an unpaired electron on the undimerized As (A). This undimerized As is the location of the surface state, $\mathrm{V} 1$. The new $\mathrm{C} 1$ is located at the empty orbitals of $\mathrm{B}$ and $\mathrm{C}$ that previously bonded toward the direction of the defect.

Next, we look in depth at how $\left(\mathrm{NH}_{4}\right)_{2} \mathrm{~S}$ and thiols react with the $\beta 2(2 \times 4)$-Defect $\mathrm{D}$ surface. $\left(\mathrm{NH}_{4}\right)_{2} \mathrm{~S}$ forms $\mathrm{S}$ in the reaction: $\left(\mathrm{NH}_{4}\right)_{2} \mathrm{~S} \rightarrow 2 \mathrm{NH}_{3}+\mathrm{H}_{2}+\mathrm{S}$. Thiols, such as ethanethiols $\left(\mathrm{CH}_{3} \mathrm{CH}_{2} \mathrm{SH}\right)$ form self-assembled monolayers ${ }^{11}$ (SAM) on the surface as follows: $\mathrm{CH}_{3} \mathrm{CH}_{2} \mathrm{SH} \rightarrow 1 / 2 \mathrm{H}_{2}$ $+\mathrm{CH}_{3} \mathrm{CH}_{2} \mathrm{~S}^{\circ}$. Figure 4 illustrates the $\beta 2(2 \times 4)$ Defect D structure and the different $\mathrm{Ga}$ and As sites on the surface. We have calculated the binding energy and replacement energy of $\mathrm{S}$ and $\mathrm{CH}_{3} \mathrm{CH}_{2} \mathrm{~S}^{\bullet}$ on this surface, referencing the As, Ga, and $\mathrm{S}$ bulk phase chemical potentials. When comparing binding and replacement at various sites, ${ }^{31}$ we find that it is energetically more favorable for sulfur (from $\left(\mathrm{NH}_{4}\right)_{2} \mathrm{~S}$ ) to replace rather than to bind on the surface at replacement site A $(2.09 \mathrm{eV})$. For $\mathrm{CH}_{3} \mathrm{CH}_{2} \mathrm{~S}^{*}$, the highest binding energy site is at the two-fold site $\mathrm{AB}(1.67 \mathrm{eV})$. 


\section{Band Structure: $\beta 2(2 \times 4)-$ Defect D}

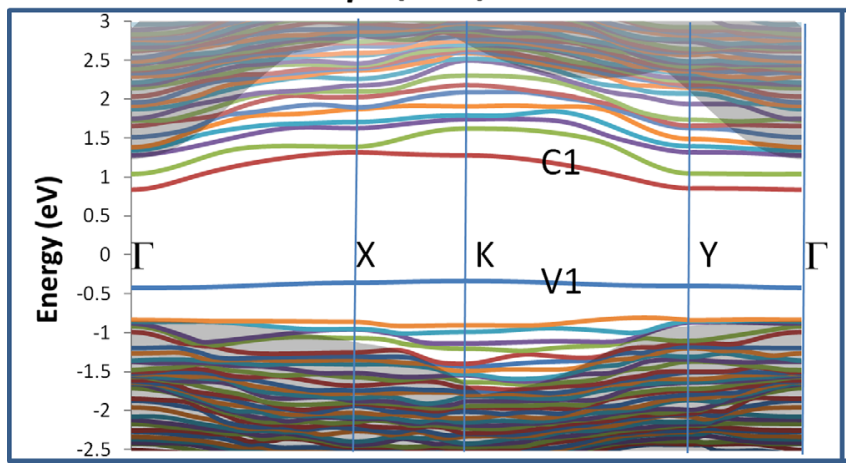

Density of States

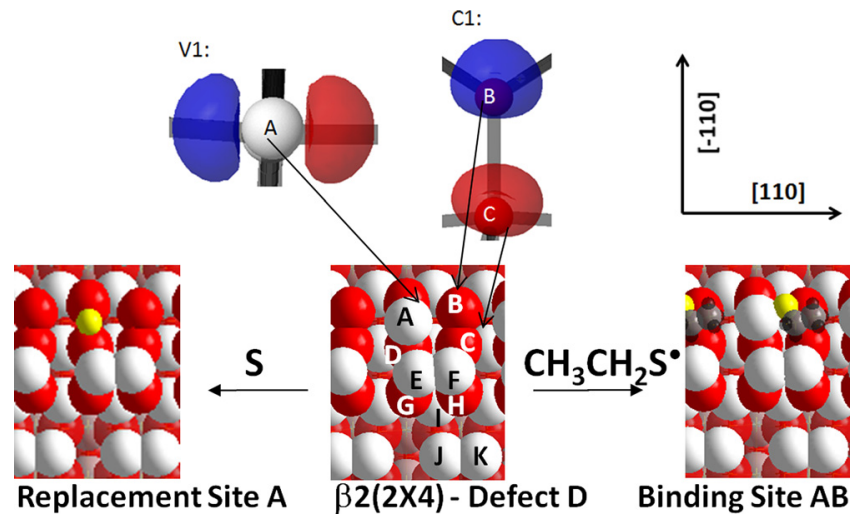

FIG. 4. Orbital visualization (blue (-) and red (+)) of the surface state, V1, and the CBM state, $\mathrm{C} 1$ of the $\beta 2(2 \times 4)$-Defect D structure. Energetically, sulfur prefers to replace at site A with a replacement energy of $2.09 \mathrm{eV}$. $\mathrm{CH}_{3} \mathrm{CH}_{2} \mathrm{~S}^{*}$ prefers to bind at the two-fold site $\mathrm{AB}$ (binding energy, $1.67 \mathrm{eV}$ ). Both reactions remove the midgap surface state, V1. White, red, yellow, black, and gray atoms represent $\mathrm{As}, \mathrm{Ga}, \mathrm{S}, \mathrm{H}$, and $\mathrm{C}$ atoms, respectively.

The substitution of sulfur at replacement site A restores the band gap and removes the midgap surface state, V1. ${ }^{31}$ Sulfur, with one more valence electron than As, pairs the undimerized electron and pushes the $\mathrm{V} 1$ state below the band gap. The $\mathrm{C} 1$ state remains. The binding of $\mathrm{CH}_{3} \mathrm{CH}_{2} \mathrm{~S}^{\circ}$ at the two-fold site $\mathrm{AB}$ pairs the undimerized electron on $\mathrm{As}$ (A) and pushes the V1 state below the band gap. ${ }^{31}$ This removes the midgap surface state and restores the band gap. Because of the bond formed between $\mathrm{CH}_{3} \mathrm{CH}_{2} \mathrm{~S}^{\circ}$ and $\mathrm{Ga}(\mathrm{B})$, the $\mathrm{C} 1$ state on atom $\mathrm{B}$ slightly delocalizes to atoms $\mathrm{G}, \mathrm{H}$, and I.

In conclusion, our experimental and theoretical results explain the defect nature of surface states and how the effectiveness of surface passivation varies for different surfaces. Our experimental results show that sulfur passivation improves the conversion efficiency of the GaAs (100) surface but not the GaAs (111B) surface. To explain this, we find with DFT calculations that the GaAs $(100) \beta 2(2 \times 4)$ surface has a cost of only $0.84 \mathrm{eV}$ to remove an As atom from its top layer, thus forming a surprisingly stable defect surface reconstruction. In contrast, the GaAs (111B) surface has a higher cost of $1.45 \mathrm{eV}$ to remove an As atom from its surface. This removal of an As atom from the surface forms a defect surface that has a midgap surface state, as shown in the band structure. During sulfur passivation, we show where $\left(\mathrm{NH}_{4}\right)_{2} \mathrm{~S}$ and $\mathrm{CH}_{3} \mathrm{CH}_{2} \mathrm{SH}$ prefer to react with the GaAs defect surface to remove the midgap surface state. Even after L2125 (1988). L1331 (1988). 116(4), 2891 (2012).

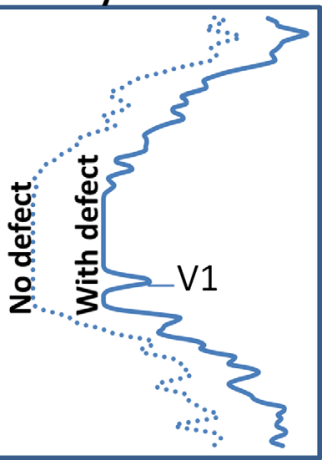

FIG. 3. The left panel represents the band structure of the $\beta 2(2 \times 4)$-Defect $D$ surface. The shaded area represents GaAs bulk states. The right panel represents the corresponding DOS. The dashed line is for a surface without a defect, the solid line for a surface with a defect. V1 and $\mathrm{C} 1$ represent the surface state and CBM.

the sulfur passivation, the GaAs (100) surface still shows lower solar conversion efficiency than the GaAs (111B) surface. This may be due to the inefficiency of the sulfur passivation process and not all of the surface states having been removed. Based on the results of this paper, with everything else being equal, we speculate that a GaAs (100) surface with the $\mathrm{c}(4 \times 4)$ reconstruction would have less surface states and improved conversion efficiency compared to the $\beta 2(2 \times 4)$ reconstruction.

This work was funded by the National Science Foundation under Grant DMR-1125931. We would like to thank participants of the IPAM program on "Materials Defects: Mathematics, Computation, and Engineering" for fruitful discussions, especially Peter Kratzer, Jörg Neugebauer, and Joshua Shapiro. We appreciate the FHIAIMS team for help with the FHI-AIMS code, with special thanks to Volker Blum and Sergey Levchenko. Special thanks to Joseph Papac for help in reviewing this manuscript.

${ }^{1}$ G. Mariani, A. C. Scofield, C. H. Hung, and D. L. Huffaker, Nat. Commun. 4, 1497 (2013).

${ }^{2}$ J. V. Holm, H. I. Jorgensen, P. Krogstrup, J. Nygard, H. Liu, and M. Aagesen, Nat. Commun. 4, 1498 (2013).

${ }^{3}$ S. Hu, Y. Kawamura, K. C. Huang, Y. Li, A. F. Marshall, K. M. Itoh, M. L. Brongersma, and P. C. Mcintyre, Nano Lett. 12(3), 1385 (2012).

${ }^{4}$ G. Mariani, P. S. Wong, A. M. Katzenmeyer, F. Leonard, J. Shapiro, and D. L. Huffaker, Nano Lett. 11(6), 2490 (2011).

${ }^{5}$ Y. Dan, K. Seo, K. Takei, J. H. Meza, A. Javey, and K. B. Crozier, Nano Lett. 11, 2527 (2011).

${ }^{6}$ G. Mariani, R. B. Laghumavarapu, B. T. de Villers, J. Shapiro, P. Senanayake, A. Lin, B. J. Schwartz, and D. L. Huffaker, Appl. Phys. Lett. 97(1), 013107 (2010).

${ }^{7}$ J. M. Jin, M. W. C. Dharmawardana, D. J. Lockwood, G. C. Aers, Z. H. Lu, and L. J. Lewis, Phys. Rev. Lett. 75(5), 878 (1995).

${ }^{8}$ C. J. Sandroff, R. N. Nottenburg, J. C. Bischoff, and R. Bhat, Appl. Phys. Lett. 51(1), 33 (1987).

${ }^{9}$ J. F. Fan, H. Oigawa, and Y. Nannichi, Jpn. J. Appl. Phys., Part 2 27(11),

${ }^{10}$ J. F. Fan, H. Oigawa, and Y. Nannichi, Jpn. J. Appl. Phys., Part 2 27(7),

${ }^{11}$ P. Arudra, G. M. Marshall, N. Liu, and J. J. Dubowski, J. Phys. Chem. C

${ }^{12}$ M. H. Sun, H. J. Joyce, Q. Gao, H. H. Tan, C. Jagadish, and C. Z. Ning, Nano Lett. 12(7), 3378 (2012).

${ }^{13}$ A. J. Howard, C. I. H. Ashby, J. A. Lott, R. P. Schneider, and R. F. Corless, J. Vac. Sci. Technol. A 12 (4), 1063 (1994).

${ }^{14}$ H. Oigawa, J. F. Fan, Y. Nannichi, K. Ando, K. Saiki, and A. Koma, Jpn. J. Appl. Phys., Part 2 28(3), L340 (1989).

${ }^{15}$ H. Ohno, H. Kawanishi, Y. Akagi, Y. Nakajima, and T. Hijikata, Jpn. J. Appl. Phys., Part 1 29(11), 2473 (1990).

${ }^{16}$ K. N. Ow and X. W. Wang, Phys. Rev. B 54(24), 17661 (1996).

${ }^{17}$ G. Hirsch, P. Kruger, and J. Pollmann, Surf. Sci. 402(1-3), 778 (1998). 
${ }^{18}$ M. Sugiyama, S. Maeyama, and M. Oshima, Phys. Rev. B 50(7), 4905 (1994).

${ }^{19}$ Y. Ke, S. Milano, X. W. Wang, N. Tao, and Y. Darici, Surf. Sci. 415, 29 (1998).

${ }^{20}$ H. Oigawa, J. F. Fan, Y. Nannichi, H. Sugahara, and M. Oshima, Jpn. J. Appl. Phys., Part 2 30(3A), L322 (1991).

${ }^{21}$ D. Usanmaz, G. P. Srivastava, and M. Cakmak, J. Appl. Phys. 108(6), 063713 (2010)

${ }^{22}$ Y. Nannichi, J. F. Fan, H. Oigawa, and A. Koma, Jpn. J. Appl. Phys., Part 2 27(12), L2367 (1988).

${ }^{23}$ W. E. Spicer, P. W. Chye, P. R. Skeath, C. Y. Su, and I. Lindau, J. Vac. Sci. Technol. 16(5), 1422 (1979).

${ }^{24}$ W. C. Wang, G. Lee, M. Huang, R. M. Wallace, and K. Cho, Microelectron. Eng. 88(12), 3419 (2011).

${ }^{25}$ W. C. Wang, G. Lee, M. Huang, R. M. Wallace, and K. J. Cho, J. Appl. Phys. 107(10), 103720 (2010).

${ }^{26}$ W. C. Wang, K. Xiong, G. Lee, M. Huang, R. M. Wallace, and K. Cho, Appl. Surf. Sci. 256(22), 6569 (2010).

${ }^{27}$ E. A. Chagarov and A. C. Kummel, Surf. Sci. 603(21), 3191 (2009).

${ }^{28}$ L. Lin and J. Robertson, Appl. Phys. Lett. 98(8), 082903 (2011).

${ }^{29}$ J. Robertson and L. Lin, Appl. Phys. Lett. 99(22), 222906 (2011).

${ }^{30}$ L. Lin and J. Robertson, J. Vac. Sci. Technol. B 30(4), 04E101 (2012).

${ }^{31}$ See supplementary material at http://dx.doi.org/10.1063/1.4826480 for experimental details, J-V curves, binding energies, and additional band diagrams.

${ }^{32}$ V. Blum, R. Gehrke, F. Hanke, P. Havu, V. Havu, X. G. Ren, K. Reuter, and M. Scheffler, Comput. Phys. Commun. 180(11), 2175 (2009).
${ }^{33}$ J. P. Perdew, K. Burke, and M. Ernzerhof, Phys. Rev. Lett. 77(18), 3865 (1996).

${ }^{34}$ A. Tkatchenko and M. Scheffler, Phys. Rev. Lett. 102(7), 073005 (2009).

${ }^{35}$ J. Heyd, G. E. Scuseria, and M. Ernzerhof, J. Chem. Phys. 124(21), 219906 (2006).

${ }^{36}$ M. Elallali, C. B. Sorensen, E. Veje, and P. Tidemandpetersson, Phys. Rev. B 48(7), 4398 (1993).

${ }^{37}$ Using this optimized GaAs lattice parameter, we calculate the bulk band gap to be $1.07 \mathrm{eV}$.

${ }^{38}$ The band gap is not converged and increasing the thickness greater than five bilayers will incrementally decrease the band gap toward the bulk value. However, due to calculation constraints of large systems, the five bi-layer slab is sufficient, as we are more interested in the qualitative appearance/disappearance of the mid-gap surface state than the exact quantitative band gap values.

${ }^{39}$ J. E. Northrup and S. Froyen, Phys. Rev. Lett. 71, 2276 (1993).

${ }^{40}$ N. Moll, A. Kley, E. Pehlke, and M. Scheffler, Phys. Rev. B 54(12), 8844 (1996).

${ }^{41}$ H. Koga, Phys. Rev. B 82(11), 113301 (2010).

${ }^{42}$ S. H. Lee, W. Moritz, and M. Scheffler, Phys. Rev. Lett. 85(18), 3890 (2000).

${ }^{43}$ With the all-electron code, we find that PBE and PBE-VdW results show $\alpha 2(2 \times 4)$ to be more favorable. Local-density approximation (LDA) and LDA-VdW results show $\zeta(4 \times 2)$ to be more favorable.

${ }^{44}$ K. Kunc and R. M. Martin, Phys. Rev. B 24(6), 3445 (1981).

${ }^{45}$ H. Yamaguchi and Y. Horikoshi, Phys. Rev. B 51(15), 9836 (1995). 